\title{
Physical activity in relation to circulating hormone concentrations in 117,100 men in UK Biobank
}

\author{
Eleanor L. Watts ${ }^{1} \mathbb{D}$ - Aurora Perez-Cornago ${ }^{1}$ - Aiden Doherty ${ }^{2,3,4} \cdot$ Naomi E. Allen $^{5,6} \cdot$ Georgina K. Fensom $^{1}$. \\ Sandar Tin Tin ${ }^{1} \cdot$ Timothy J. Key ${ }^{1} \cdot$ Ruth C. Travis ${ }^{1}$
}

Received: 18 January 2021 / Accepted: 14 June 2021 / Published online: 3 July 2021

(c) The Author(s) 2021

\begin{abstract}
Purpose Physical activity may reduce the risk of some types of cancer in men. Biological mechanisms may involve changes in hormone concentrations; however, this relationship is not well established. Therefore, we aimed to investigate the associations of physical activity with circulating insulin-like growth factor-I (IGF-I), sex hormone-binding globulin (SHBG, which modifies sex hormone activity), and total and free testosterone concentrations, and the extent these associations might be mediated by body mass index (BMI).

Methods Circulating concentrations of these hormones and anthropometric measurements and self-reported physical activity data were available for 117,100 healthy male UK Biobank participants at recruitment. Objectively measured accelerometer physical activity levels were also collected on average 5.7 years after recruitment in 28,000 men. Geometric means of hormone concentrations were estimated using multivariable-adjusted analysis of variance, with and without adjustment for BMI. Results The associations between physical activity and hormones were modest and similar for objectively measured (accelerometer) and self-reported physical activity. Compared to men with the lowest objectively measured physical activity, men with high physical activity levels had 14\% and $8 \%$ higher concentrations of SHBG and total testosterone, respectively, and these differences were attenuated to $6 \%$ and $3 \%$ following adjustment for BMI.

Conclusion Our results suggest that the associations of physical activity with the hormones investigated are, at most, modest; and following adjustment for BMI, the small associations with SHBG and total testosterone were largely attenuated. Therefore, it is unlikely that changes in these circulating hormones explain the associations of physical activity with risk of cancer either independently or via BMI.
\end{abstract}

Keywords Physical activity $\cdot$ Testosterone $\cdot$ IGF-I $\cdot$ SHBG $\cdot$ Accelerometer $\cdot$ UK Biobank

Eleanor L. Watts

ellie.watts@ndph.ox.ac.uk

1 Cancer Epidemiology Unit, Nuffield Department of Population Health, University of Oxford, Richard Doll Building, Old Road Campus, Oxford OX3 7LF, UK

2 Big Data Institute, Li Ka Shing Centre for Health Information and Discovery, University of Oxford, Oxford, UK

3 Nuffield Department of Population Health, University of Oxford, Oxford, UK

4 Clinical Trial Service Unit and Epidemiological Studies Unit, Nuffield Department of Population Health, University of Oxford, Oxford, UK

5 UK Biobank Ltd, Cheadle, Stockport, UK

6 National Institute of Health Research Oxford Biomedical Research Centre, Oxford, UK

$\begin{array}{ll}\text { Abbreviations } \\ \text { ANOVA } & \text { Analysis of variance } \\ \text { BMI } & \text { Body mass index } \\ \text { CI } & \text { Confidence interval } \\ \text { IGF-I } & \text { Insulin-like growth factor-I } \\ \text { IPAQ } & \text { International Physical Activity Questionnaire } \\ \text { IQR } & \text { Interquartile range } \\ \text { MET } & \text { Metabolic equivalents of task } \\ \text { SD } & \text { Standard deviation } \\ \text { SHBG } & \text { Sex hormone-binding globulin } \\ \text { WHR } & \text { Waist-to-hip ratio }\end{array}$

Abbreviations

ANOVA Analysis of variance

BMI Body mass index

CI Confidence interval

IGF-I Insulin-like growth factor-I

IPAQ International Physical Activity Questionnaire

IQR Interquartile range

MET Metabolic equivalents of task

SD Standard deviation

WHR Waist-to-hip ratio 


\section{Introduction}

Higher physical activity levels are associated with a lower risk of some types of cancer in men [1]. Physical activity may be associated with cancer risk via changes in hormone concentrations, metabolism, immune function, body composition and/ or by reducing low-grade inflammation [2-4]. However, clear biological mechanisms linking physical activity to cancer risk are not well established. There is consistent epidemiological evidence that the hormones insulin-like growth factor-I (IGFI), sex hormone-binding globulin (SHBG: this molecule is not a hormone, but modifies sex hormone activity), and total and free testosterone may be related to cancer risk. In particular, higher IGF-I concentration has been associated with an increased risk of prostate, thyroid and colorectal cancers [5-8], while higher free testosterone has been associated with an increased risk of prostate cancer and possibly malignant melanoma in men [7, 9]. Previous studies investigating the possible effect of physical activity on serum hormone concentrations in men have been inconclusive [10-19]. However, observational studies were generally based on self-reported physical activity, with a limited sample size (up to 6,000) [10-13]. Randomised controlled trials generally have been of limited duration, potentially biased by participant dropout, and may have been underpowered to detect more modest associations [14-19]. Body mass index (BMI) also has well-established associations with IGF-I, SHBG, total and free testosterone concentrations [20, $21]$, and it is not clear whether, and to what extent, physical activity is associated with circulating hormone concentrations independently of BMI.

In this paper, we aimed to examine the associations of objectively measured and self-reported physical activity and sedentary time, as well as anthropometric factors, with serum concentrations of IGF-I, SHBG, and total and free testosterone in a cohort of 117,100 men; we recently reported similar analyses in women [22]. As adiposity may be important in the associations between physical activity and hormones, we first describe the associations of BMI with the hormones, and then the associations of physical activity with the hormones both without and with adjustment for BMI.

\section{Materials and methods}

The design of the analyses, presentation and description are in accordance with the STROBE checklist [23].

\section{Study design}

UK Biobank is a prospective cohort for public health research. Details of the study protocol and data collection are available online (http://www.ukbiobank.ac.uk/wp-conte
nt/uploads/2011/11/UK-Biobank-Protocol.pdf) and elsewhere [24, 25].

In brief, all participants were registered with the UK National Health Service and lived within $40 \mathrm{~km}$ of one of the UK Biobank assessment centres. Approximately 9.2 million people were initially invited to participate. Overall, 503,317 participants $(229,122$ men) aged 40-69 years consented to join the cohort and attended one of 22 assessment centres throughout England, Wales and Scotland between 2006 and 2010, a participation rate of 5.5\% [25].

The UK Biobank study was approved by the North West Multi-Centre Research Ethics Committee (reference number 06/MRE08/65), and at recruitment, all participants gave written informed consent to participate and for their health to be followed-up through linkage to electronic medical records.

\section{Baseline assessment}

At the baseline assessment visit, participants provided information on a range of sociodemographic, anthropometric, lifestyle, and health-related factors via a self-completed touch-screen questionnaire and a computer-assisted personal interview [25].

\section{Anthropometry}

Anthropometric measurements were taken at the assessment centre at study baseline [25]. Height was measured using the Seca 202 height measure (Seca, Hamburg, Germany), and waist and hip measurements were made over light clothes with the Seca-200 tape measure (Seca, Hamburg, Germany). Weight and bio-impedance measures were taken using the Tanita BC418MA body composition analyser (Tanita, Tokyo, Japan). BMI was calculated as weight $(\mathrm{kg}) / \mathrm{height}$ $(\mathrm{m})^{2}$. Comparative body size and height at age 10 were selfreported via the touch-screen questionnaire at recruitment.

\section{Self-reported physical activity}

Self-reported physical activity was assessed using questions adapted from the International Physical Activity Questionnaire (IPAQ) short form, a validated survey based on the frequency and duration of walking, moderate and vigorous activity [26].

The duration of each level of activity was weighted by estimated metabolic equivalent of tasks (METs) (3.3, 4.0 and 8.0 METs for walking, moderate intensity and vigorous intensity, respectively), to estimate total MET hours per week of physical activity. Following IPAQ guidelines, physical activity for any category of less than 10 min per day was recoded to 0 and durations of $>180 \mathrm{~min}$ per day were truncated [26]. 
Sedentary time was estimated as the total hours per week that participants reported spending watching television or using a computer (not including work).

\section{Accelerometer assessment}

Between February 2013 and December 2015, participants who provided a valid email address were selected at random to receive email invitations to wear an Axivity AX3 wrist-worn accelerometer for 7 days to objectively measure physical activity levels (44.8\% response rate) [27]. Accelerometer-measured physical activity was defined as the vector magnitude of acceleration (in milligravity units) averaged over five-second time windows [27]. This has been validated against the doubly labelled water method which is a gold standard measure for energy expenditure in participants with stable weight [28].

The percentages of time spent in moderate and vigorous, light tasks and walking physical activity as well as sedentary time measured by the accelerometer were calculated using machine learning methods that have been described in more detail elsewhere [29, 30]. These methods extracted time and frequency domain features from each $30 \mathrm{~s}$ window in the accelerometer time series. Random forests and hidden Markov models were then trained and evaluated in 153 free-living individuals (mean age $=42$, male $n=53$ ) to distinguish between activity states, evaluated against reference wearable camera, time-use and sleep diary information sources.

\section{Blood sampling and biomarker assays}

At recruitment, blood sampling was successfully performed in $99.7 \%$ of the cohort. Blood was collected in a serum separator tube and shipped to the central processing laboratory in temperature-controlled boxes at $4{ }^{\circ} \mathrm{C}$ [31], then aliquoted and stored in a central working archive at $-80{ }^{\circ} \mathrm{C}$ [32]. Measurements of serum concentrations of IGF-I, SHBG, testosterone and albumin were attempted in all participants. IGF-I was determined by chemiluminescent immunoassays (DiaSorin Liaison XL), and SHBG and testosterone concentrations were measured using chemiluminescent immunoassays (Beckman Coulter Unicel DxI 800). Albumin was measured by a colorimetric assay (Beckman Coulter AU5800). Average within-laboratory (total) coefficients of variation for low, medium and high internal quality control level samples for each biomarker ranged from 2.1-8.3\%. Full details of the assay methods and quality assurance protocols are available online (https://biobank.ndph.ox.ac.uk/showc ase/docs/serum_biochemistry.pdf).

\section{Free testosterone calculation}

In the circulation, approximately $98 \%$ testosterone is bound to SHBG and albumin. The remaining $2 \%$ circulates as unbound or "free" testosterone and is hypothesised to be biologically active [33]. Free testosterone concentrations were estimated using a formula based on the law of mass action and measured total testosterone, SHBG and albumin concentrations [7, 34].

\section{Repeat measurements}

Participants who lived within a $35-\mathrm{km}$ radius were invited via email to attend a repeat assessment clinic at the UK Biobank Co-ordinating Centre in Stockport between August 2012 and June 2013. Repeat assessments including blood sampling and self-reported physical activity and sedentary behaviour were completed in 9,000 men with a response rate of $21 \%$ [35].

\section{Exclusion criteria}

We excluded 9,869 men with prevalent cancer (except C44: non-melanoma skin cancer). We also excluded 13,524 men who did not have hormone measurements available or who had biomarker measurements that did not pass quality control procedures [36], 780 men who had no BMI data. Chronic illness and diabetes is associated with altered hormone concentrations [37] and also may affect engagement in physical activity; therefore, we excluded 15,074 men who reported having diabetes and 48,217 men who reported being in poor or fair overall health. We also excluded 1,226 participants for whom it was not possible to determine genetic sex or who were identified as being genetically female, and 1,290 men who reported taking hormone medication at baseline (Supplementary Figure S1).

For the analysis of accelerometer data, we also excluded 1,190 men who were diagnosed with cancer between baseline and accelerometer reading. We excluded 2,064 men with insufficient wear time, poor calibration, $>1 \%$ clipped values (which occur when the sensor's dynamic range of $+-8 \mathrm{~g}$ is exceeded before or after calibration), and participants with implausibly high activity values, as described elsewhere [27]. For analysis of self-reported physical activity data, we excluded 21,543 men with missing or incomplete physical activity data and 449 men who reported undertaking more than $16 \mathrm{~h}$ of physical activity per day [26].

In total, our analytical dataset included 117,143 healthy men with valid self-reported physical activity and 27,933 with accelerometer measurements (Supplementary Figure S1). 


\section{Statistical analysis}

IGF-I, SHBG and total and free testosterone concentrations were logarithmically transformed to approximate a normal distribution. Analyses examined associations with anthropometric factors, accelerometer-measured physical activity and self-reported physical activity. The anthropometric factors were BMI $(<22.5, \geq 22.5-<25, \geq 25-<27.5, \geq 27.5-<30$. $\left.0, \geq 30.0-<35.0, \geq 35 \mathrm{~kg} / \mathrm{m}^{2}\right)$, height $(<170, \geq 170-<175$, $\geq 175-<180, \geq 180-<185, \geq 185 \mathrm{~cm})$, waist circumference (cm, fifths), waist-to-hip ratio (WHR) (fifths), body size aged 10 (thinner, about average, plumper), height aged 10 (shorter, about average, taller). Measured physical activity variables were overall score (milligravity, fifths), moderate physical activity (\% time spent, fifths), light tasks (\% time spent, fifths), walking (\% time spent, fifths) and sedentary (\% time spent, fifths). Self-reported physical activity variables were total METs (hours per week, fifths), vigorous (hours per week, fifths), moderate (hours per week, fifths), walking (hours per week, fifths) and sedentary time (hours per week, fifths).

Geometric mean hormone concentrations were calculated using predicted values from analysis of variance (ANOVA) models scaled to the overall geometric mean concentration and adjusted for age at recruitment $(<45, \geq 45-<49$ $, \geq 50-<54, \geq 55-<59, \geq 60-<64, \geq 65$ years), geographic area (10 UK regions), Townsend deprivation score (fifths, unknown $(0.1 \%)$ ), racial/ethnic group (white, mixed background, Asian, black, other and unknown $(0.5 \%))$, height (categories as above), cigarette smoking (never, former, current light smoker $(1-<15$ cigarettes per day), current heavy smoker ( $\geq 15$ cigarettes per day), current (number of cigarettes per day unknown), and smoking status unknown (0.6\%)), and alcohol consumption (non-drinkers, $<1-<10, \geq 10-<20, \geq 20 \mathrm{~g}$ ethanol/day, unknown $(0.5 \%))$. In analyses of height as the exposure, height was not included as an adjustment factor and in analyses of physical activity. The primary model was also further adjusted for BMI (categories as above). Adjustment covariates were defined a priori based on previous analyses of UK Biobank data $[22,38]$, and categories were used to account for nonlinear associations.

Heterogeneity of means by category was tested using the $\mathrm{F}$ test. $\mathrm{P}_{\text {trend }}$ was estimated using the ANOVA test with the categorical variables entered as linear values scored consecutively as the median values within each quantile.

\section{Further analyses}

To compare the magnitudes of the associations of anthropometric and physical activity measures with hormone concentrations, and the role of possible confounders in the associations, we estimated percentage change in hormone concentration per $1 \mathrm{SD}$ increase using minimally adjusted (adjusted for age and region categories as defined above) and multivariable-adjusted ANOVA models with standardised continuous exposure variables.

Repeat hormone concentrations and accelerometer scores were available in a subset of up to 2,372 men, and repeat measures of both hormones and self-reported physical activity were available in up to $6,027 \mathrm{men}$. To assess the robustness of our results, analyses were repeated using (i) the single accelerometer score and mean baseline and repeat hormone measurements, and (ii) the means of baseline and repeat assessment values for both self-reported physical activity and hormone measurements. These associations were compared to the baseline only associations in this subset.

In further analyses, we tested for heterogeneity by the possible effect modifiers: (i) BMI category $(<25, \geq 25$ $<27.5, \geq 27.5-<30, \geq 30 \mathrm{~kg} / \mathrm{m}^{2}$ ), (ii) employment status (employed or self-employed vs not employed or selfemployed), (iii) regularity of heavy manual or physical work (sometimes/usually/always vs never) using the likelihood ratio test. To examine the shape of the associations, we also repeated the primary analysis examining associations with BMI and physical activity in deciles.

All analyses were performed using Stata version 14.1 (Stata Corporation, College Station, TX, USA), and figures were plotted in $\mathrm{R}$ version 3.2.3. All tests of significance were two-sided, and $\mathrm{P}$ values $<0.05$ were considered statistically significant.

\section{Results}

In total, 117,143 and 27,933 men were included in this analysis with valid self-reported and accelerometer-measured physical activity, respectively. Men with accelerometermeasured physical activity data were on average 56.3 years old at recruitment (standard deviation $(\mathrm{SD})=7.9$ ), 97\% were white, $7 \%$ were current smokers, and mean BMI was $26.7 \mathrm{~kg} / \mathrm{m}^{2}(\mathrm{SD}=3.5)$ (Table 1$)$. Median accelerometer-measured physical activity score was 27.4 milligravity $(\mathrm{IQR}=10.2)$. In men with valid self-reported physical activity levels, median METs were $33.9 \mathrm{~h}$ per week (interquartile range $(I Q R)=49.9)$ (Table 1). Men who took part in the accelerometer study were slightly more likely to have a university degree and were less likely to be current smokers than men with valid self-reported physical activity estimates (Table 1).

Participants with higher accelerometer measurements were on average younger, had a lower BMI, were less likely to be current smokers and more likely to be in paid employment or self-employed. These men also had higher levels of self-reported vigorous, moderate and walking physical 
Table 1 Participant characteristics in UK Biobank male participants with valid self-reported physical activity and accelerometer data

\begin{tabular}{|c|c|c|}
\hline & $\begin{array}{l}\text { Accelerometer } \\
(n=27,933)\end{array}$ & $\begin{array}{l}\text { Self-reported physica } \\
\text { activity }(n=117,143)\end{array}$ \\
\hline \multicolumn{3}{|l|}{ Sociodemographic factors } \\
\hline Age at recruitment (years), mean (SD) & $56.3(7.9)$ & $56.2(8.2)$ \\
\hline White, $n(\%)$ & $27,128(97.4)$ & $112,117(96.0)$ \\
\hline Townsend deprivation score, med (IQR) & $-2.6(3.4)$ & $-2.5(3.6)$ \\
\hline University degree, $n(\%)$ & $21,336(82.5)$ & $82,313(79.7)$ \\
\hline Paid employment/self-employed, $n(\%)$ & $18,621(66.7)$ & $77,881(66.5)$ \\
\hline Current smoker, $n(\%)$ & $1,961(7.0)$ & $11,172(9.6)$ \\
\hline Alcohol ( $\geq 10 \mathrm{~g}$ ethanol per day), $n(\%)$ & $19,029(68.2)$ & $80,115(68.5)$ \\
\hline \multicolumn{3}{|l|}{ Health-related factors } \\
\hline Vasectomy, $n(\%)$ & $1,725(6.2)$ & $6,734(5.7)$ \\
\hline Hypertensive, $n(\%)$ & $13,634(48.8)$ & $59,269(50.6)$ \\
\hline Family history of prostate cancer, $n(\%)$ & $2,255(13.6)$ & $9,029(13.2)$ \\
\hline \multicolumn{3}{|l|}{ Anthropometric factors } \\
\hline BMI $\left(\mathrm{kg} / \mathrm{m}^{2}\right)$, mean $(\mathrm{SD})$ & $26.7(3.5)$ & $27.1(3.6)$ \\
\hline Height $(\mathrm{cm})$, mean $(\mathrm{SD})$ & $176.7(6.6)$ & $176.2(6.7)$ \\
\hline Waist circumference $(\mathrm{cm})$, mean $(\mathrm{SD})$ & $93.8(9.8)$ & $94.7(9.9)$ \\
\hline Waist-to-hip ratio, mean (SD) & $0.9(0.1)$ & $0.9(0.1)$ \\
\hline Body size age 10 years (plumper), $n(\%)$ & $3,490(12.7)$ & $14,305(12.4)$ \\
\hline Height age 10 years (above average), $n(\%)$ & $7,443(26.9)$ & $30,132(26.0)$ \\
\hline \multicolumn{3}{|l|}{ Accelerometer-measured physical activity } \\
\hline Overall score (milligravity), med (IQR) & $27.4(10.2)$ & - \\
\hline Moderate physical activity (hrs per week), med (IQR) & $7.0(6.7)$ & - \\
\hline Light tasks (hrs per week), med (IQR) & $7.4(6.7)$ & - \\
\hline Walking (hrs per week), med (IQR) & $18.7(9.8)$ & - \\
\hline Sedentary (hrs per week), med (IQR) & $70.8(16.3)$ & - \\
\hline \multicolumn{3}{|l|}{ Self-reported physical activity } \\
\hline METs (hrs per week), med (IQR) & $32.1(43.7)$ & $33.9(49.9)$ \\
\hline Vigorous physical activity (hrs per week), med (IQR) & $1.0(2.5)$ & $1.0(2.5)$ \\
\hline Moderate physical activity (hrs per week), med (IQR) & $2.0(4.5)$ & $2.0(4.6)$ \\
\hline Walking physical activity (hrs per week), med (IQR) & $3.0(5.5)$ & $3.5(5.3)$ \\
\hline Leisure sedentary activity (hrs per week), mean (SD) & $21.0(21.0)$ & $21.0(21.0)$ \\
\hline
\end{tabular}

${ }^{\mathrm{a}} \mathrm{Med}(\mathrm{IQR})$ displayed where data are not normally distributed

$B M I$ body mass index; IQR interquartile range; med median; $M E T$ metabolic equivalent of task; $S D$ standard deviation

activity, and spent less time sedentary (Table 2). Participants in the highest fifth of self-reported physical activity levels had a lower socioeconomic status, were less likely to be in paid employment or self-employed, had a higher overall accelerometer score and accelerometer-measured physical activity subtype values, and less sedentary time (Table 2).

The correlation between self-reported MET hours per week at baseline and repeat assessment (on average 4.3 years later) was relatively high (Spearman's $r=0.61$ ), and pairwise correlations between the biomarkers ranged from $0.85-0.55$, for SHBG and free testosterone, respectively (Supplementary Table S1). Median absolute levels of selfreported physical activity and biomarkers at baseline and repeat measure are available in Supplementary Table S1. The correlations between MET hours per week and overall accelerometer score were lower at $r=0.23$ and $r=0.28$ in the baseline and repeat sample, respectively (mean time from baseline to accelerometer measurement $=5.7$ years and mean time from repeat assessment to accelerometer measurement $=2.2$ years).

The exposure-outcome associations are described below only if there was $\geq 5 \%$ difference in hormone concentrations across the range of the exposure.

\section{Anthropometric factors}

Men with BMI $\geq 35 \mathrm{~kg} / \mathrm{m}^{2}$ had concentrations of SHBG, total testosterone, IGF-I and free testosterone that were $27 \%$, $25 \%, 12 \%$ and $12 \%$ lower, respectively, than for men with BMI $\geq 22.5-<25.0 \mathrm{~kg} / \mathrm{m}^{2}$ (Supplementary Table S2). IGF-I 


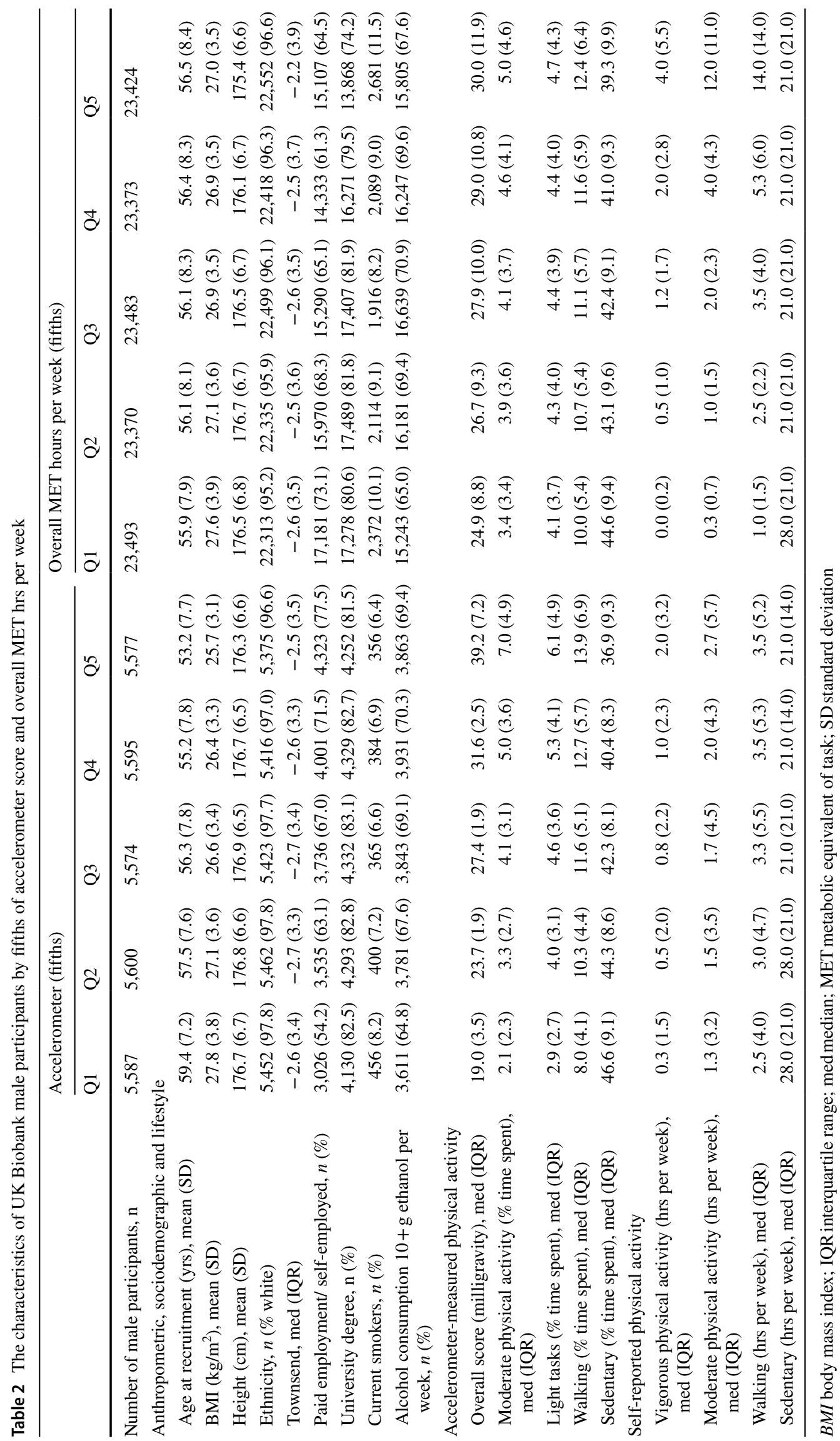


and free testosterone had inverse U-shaped associations with BMI, whereas the associations with SHBG and total testosterone were approximately linear (Fig. 1). Associations with other anthropometric factors are displayed in Supplementary Table S2.

\section{Physical activity}

\section{Accelerometer-measured physical activity}

Men in the highest fifth of overall accelerometer-measured physical activity had $14 \%$ and $8 \%$ higher concentrations of SHBG and total testosterone, respectively, in comparison with men in the lowest fifth (Fig. 1). After further adjustment for BMI, the magnitudes of the associations attenuated to $6 \%$ and $3 \%$, respectively (Table 3 ).

Men in the highest fifth of time spent doing moderate and light tasks had 10\% and 6\% higher SHBG in comparison with men in the lowest fifth, but following adjustment for BMI, the magnitudes of the associations attenuated to $4 \%$ and $2 \%$, respectively (Table 3 ). Men who spent the highest proportion of time sedentary had $8 \%$ lower SHBG, but this was attenuated to $3 \%$ lower following adjustment for BMI.

\section{Self-reported physical activity}

Men in the highest fifth of overall physical activity had $12 \%$ and $6 \%$ higher concentrations of SHBG and total testosterone, respectively, in comparison with the lowest fifth (Fig. 1). After further adjustment for BMI, the associations attenuated to $10 \%$ and $4 \%$, respectively (Fig. 1).

Men in the highest fifth of vigorous, moderate and walking physical activity categories had $12 \%, 9 \%$ and $5 \%$ higher SHBG concentrations, respectively; and following adjustment for BMI, these associations were attenuated to $10 \%$, $7 \%$ and $4 \%$, respectively. Men in the highest fifth of sedentary activity had 6\% lower concentrations of SHBG in comparison with men in the lowest fifth; following further adjustment for BMI, the magnitude of the association was attenuated to null (Table 3).

The associations of accelerometer-measured and selfreported physical activity with IGF-I and free testosterone were significant, but small (all $<2 \%$ differences between highest and lowest fifths).

\section{Further analyses}

\section{Percentage change in hormone concentration per 1 SD increase in exposure measures}

Without adjustment for BMI, the magnitudes of the associations between accelerometer-measured physical activity and serum hormone concentrations per $1 \mathrm{SD}$ increase in physical activity were generally larger than those observed with selfreported physical activity (Supplementary Table S3). Further adjustment for BMI led to attenuations of the associations with both measures of physical activity. The magnitudes of the associations with SHBG and total testosterone concentrations were generally larger for higher than for lower intensities of physical activity (whether assessed by accelerometer or self-report), both with and without adjustment for BMI. Associations in the minimally adjusted models are also displayed in Supplementary Table S3.

\section{Repeat assessment and associations in tenths}

When we restricted analyses to men who had attended the repeat assessment, and estimated associations using mean hormone and physical activity measurements, associations between physical activity and hormones were not materially different (Supplementary Table S4). The shapes of the associations were similar when we examined the relationship with BMI and physical activity in tenths (Supplementary Figure S2).

\section{Subgroup analyses}

There was evidence of heterogeneity in the associations of both measured and self-reported physical activity with IGF-I and free testosterone by category of BMI $\left(\mathrm{P}_{\text {het }}<0.01\right)$. In men with obesity, those with higher physical activity levels had higher IGF-I and free testosterone concentrations than men with lower physical activity levels, while the association of physical activity with hormones was flatter/moderately inverse in men who were not obese (Figs. 2 and 3). However, the magnitudes of the associations were small (up to 5\% elevated IGF-I/free testosterone concentrations in men with obesity in the highest fifth of physical activity in comparison with the lowest). There was also evidence of heterogeneity in the association of measured physical activity and SHBG $\left(\mathrm{P}_{\text {het }}<0.0001\right)$, with smaller magnitudes of associations observed in men with higher BMIs.

There was significant heterogeneity in the associations of self-reported and accelerometer-measured physical activity with SHBG and total testosterone by employment status. Men who were employed at study baseline had slightly larger magnitudes of associations than those who were not ( $1^{\text {st }}$ fifth vs the 5th) (Supplementary Table S5). Similar patterns of heterogeneity were present by physical activity at work, with slightly larger magnitudes of associations observed of physical activity with SHBG and total testosterone observed in men whose jobs did not involve manual labour, but these observed differences were modest (Supplementary Table S6). 

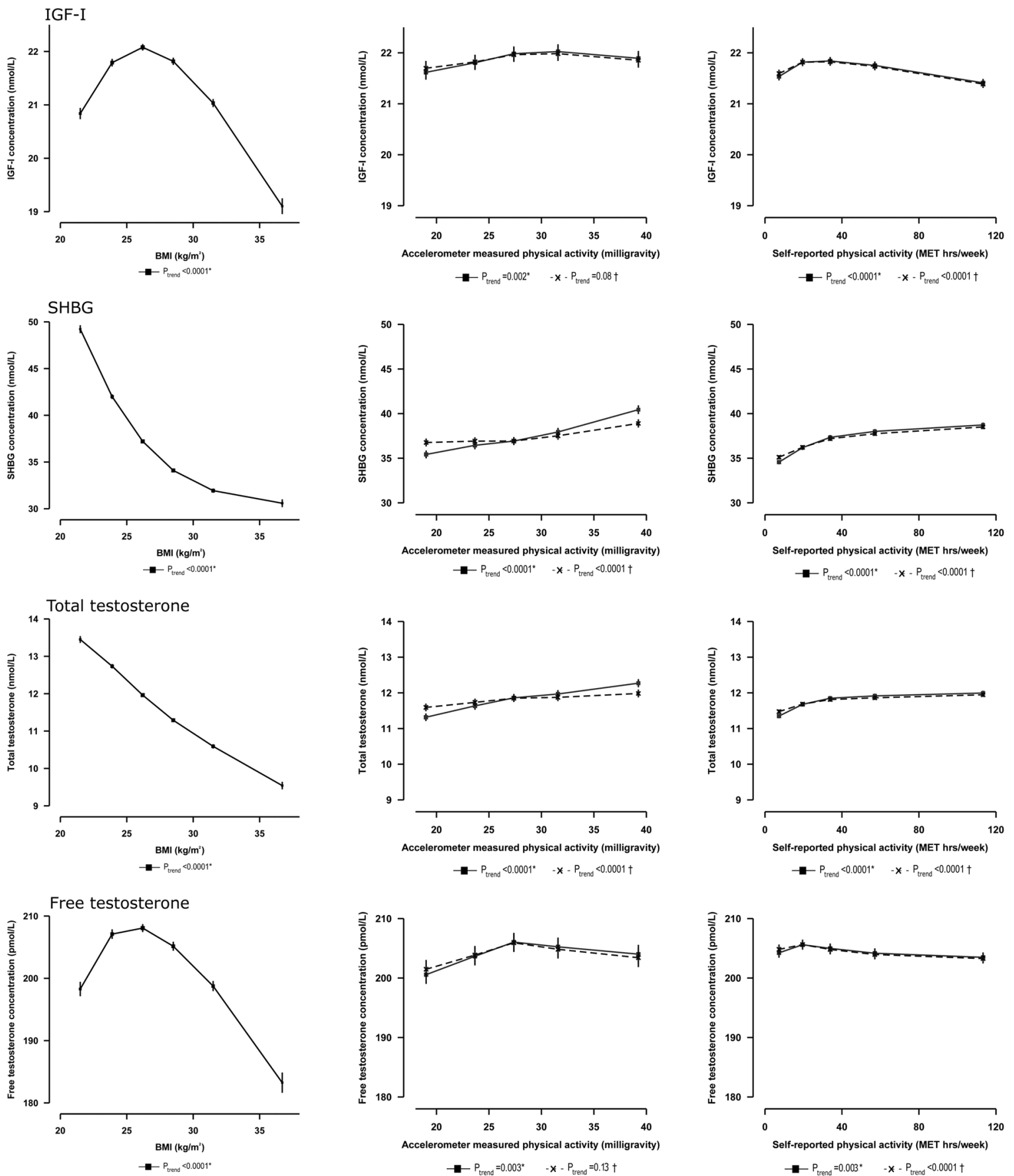

Fig. 1 Adjusted geometric mean circulating hormone concentrations by BMI, accelerometer-measured and self-reported physical activity levels, in UK Biobank male participants. Geometric mean concentrations are presented by data points plotted as the median value within each fifth, with their $95 \%$ CIs represented as horizontal lines. $\mathrm{P}_{\text {trend }}$ are estimated using the analysis of variance test, with the categorical variables entered as linear values scored consecutively as the median values within each fifth. *Model 1: Estimated geometric mean concentrations adjusted for age at recruitment, geographic area, Townsend deprivation score, racial/ethnic group, height, cigarette smoking, alcohol consumption (solid line). $†$ Model 2: Model $1+$ further adjusted for BMI (dashed line). BMI = body mass index; IGF-I = insulin-like growth factor-I; SHBG=sex hormone-binding globulin 


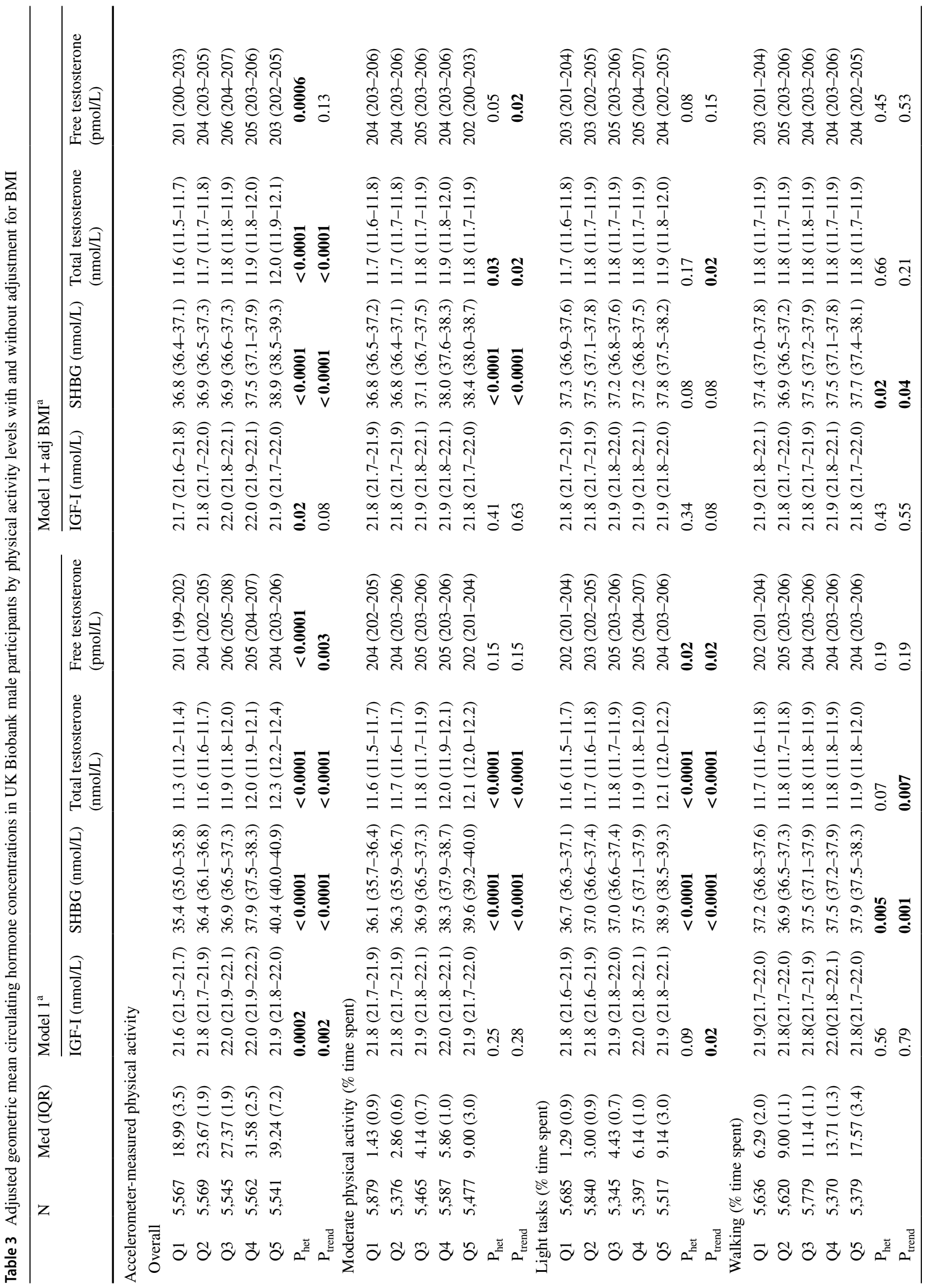




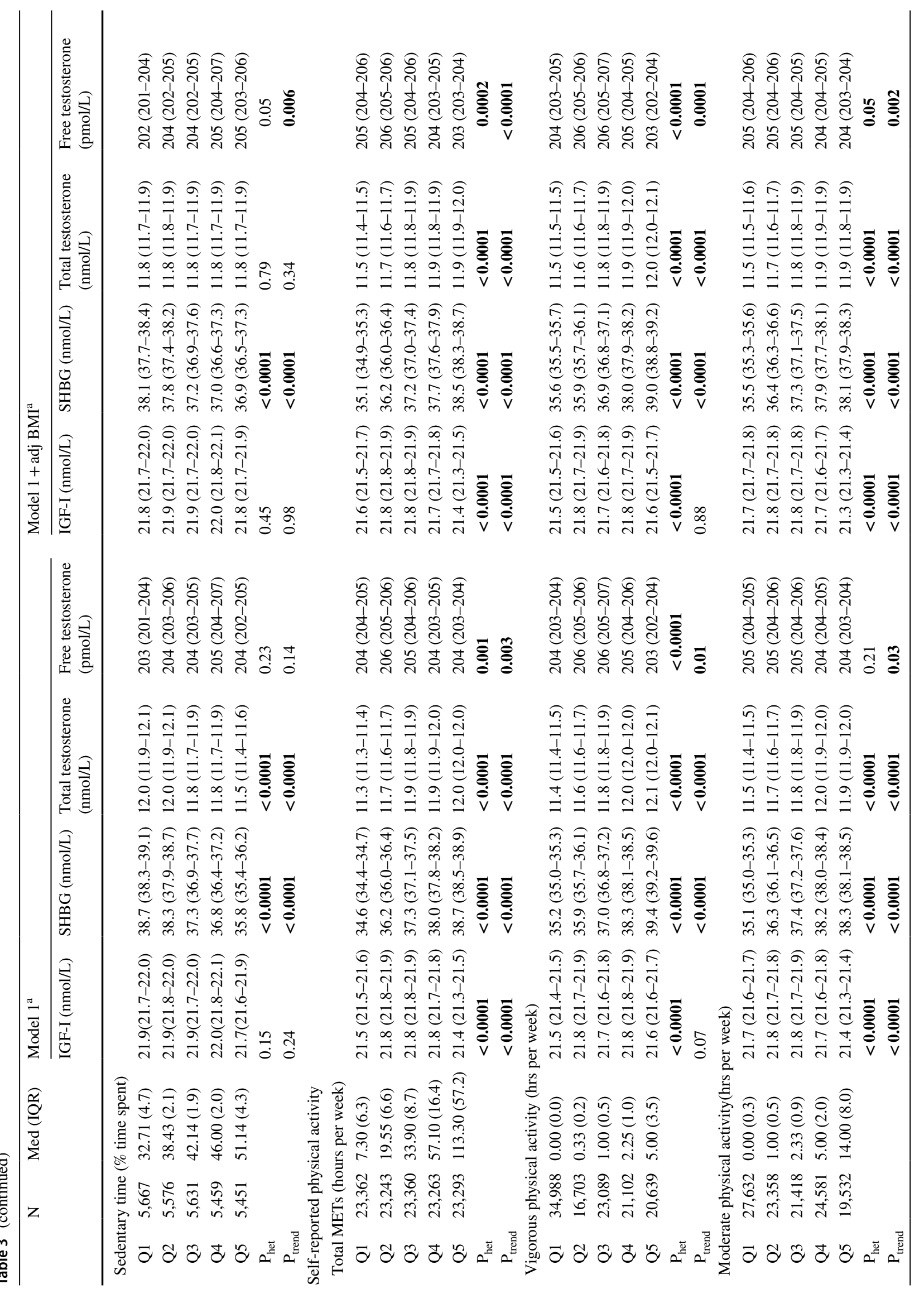




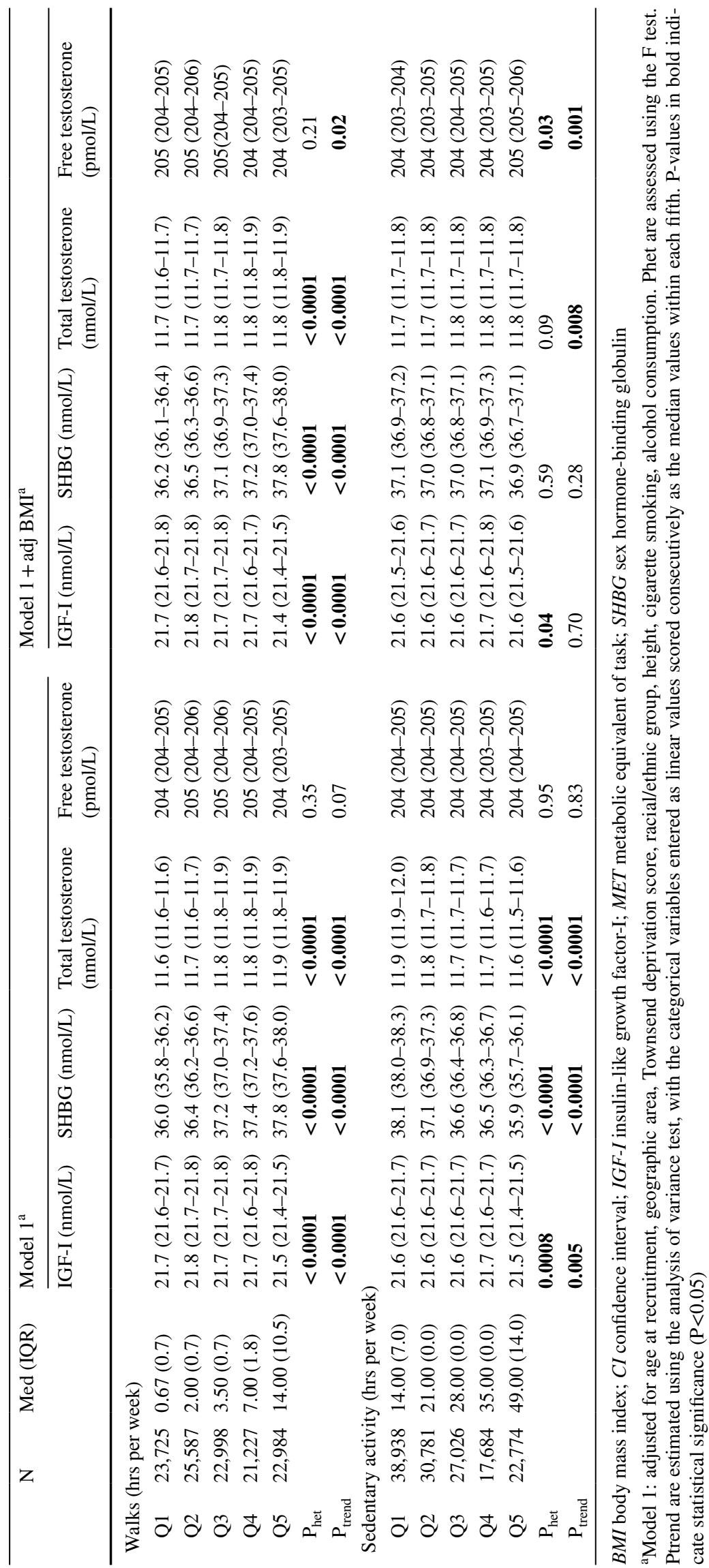



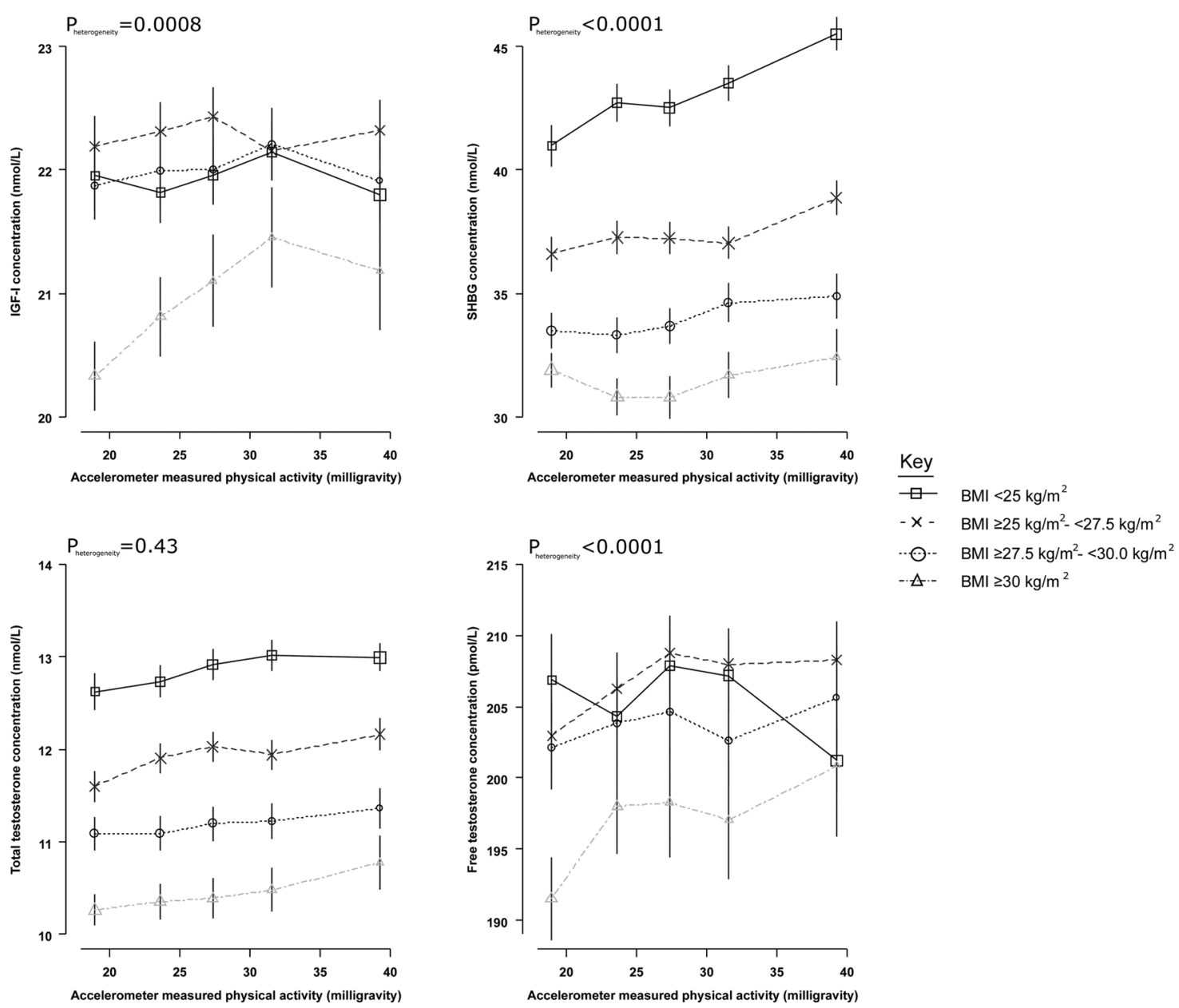

Fig. 2 Adjusted geometric mean circulating hormone concentrations by accelerometer-measured physical activity, stratified by categories of BMI, in UK Biobank male participants. Estimated geometric mean concentrations are adjusted for age at recruitment, geographic area, Townsend deprivation score, racial/ethnic group, height, ciga-

\section{Discussion}

This observational analysis of 117,100 men showed that those with higher physical activity levels had modestly elevated SHBG and higher total testosterone concentrations, while the differences in IGF-I and free testosterone by physical activity were very small and are unlikely to be biologically meaningful. Following adjustment for BMI, the small associations of physical activity with these biomarkers were largely attenuated.

Although there were statistically significant associations between measured, self-reported physical activity and sedentary time and IGF-I concentrations, the magnitudes of the associations were small (generally $\sim 1 \%$ in the highest group in comparison with the lowest), suggesting that higher physical activity is unlikely to have a meaningful impact on cancer risk mediated by IGF-I. The magnitudes of these rette smoking, alcohol consumption. Geometric mean concentrations are presented by data points plotted as the median value within each fifth, with their $95 \%$ CIs represented as horizontal lines. $\mathrm{P}_{\text {heterogeneity }}$ is assessed using the $\mathrm{F}$ test. $\mathrm{BMI}=$ body mass index; IGF-I = insulinlike growth factor-I; SHBG = sex hormone-binding globulin associations are largely consistent with other cross-sectional analyses [11, 22, 39], while results from clinical trials have been inconclusive [15-17, 40].

Physical activity may lead to elevated SHBG concentrations by reducing low-grade inflammation [41, 42], altering factors related to insulin resistance $[42,43]$ and possibly reducing liver fat [44]; these physiological changes may be due to modifications in body composition [45]. Our findings are largely consistent with results from clinical trials [14, 46], and some [12, 22, 47], but not all [13], cross-sectional analyses. The slightly elevated testosterone concentrations in men with higher levels of physical activity may be due to elevated SHBG concentrations, which increases the half-life of testosterone and may also result in an increase in testosterone production via the hypothalamic-pituitary-gonadal axis [48]. Findings from other studies are inconclusive $[10,12-15,18,46]$, but power was generally limited. In 

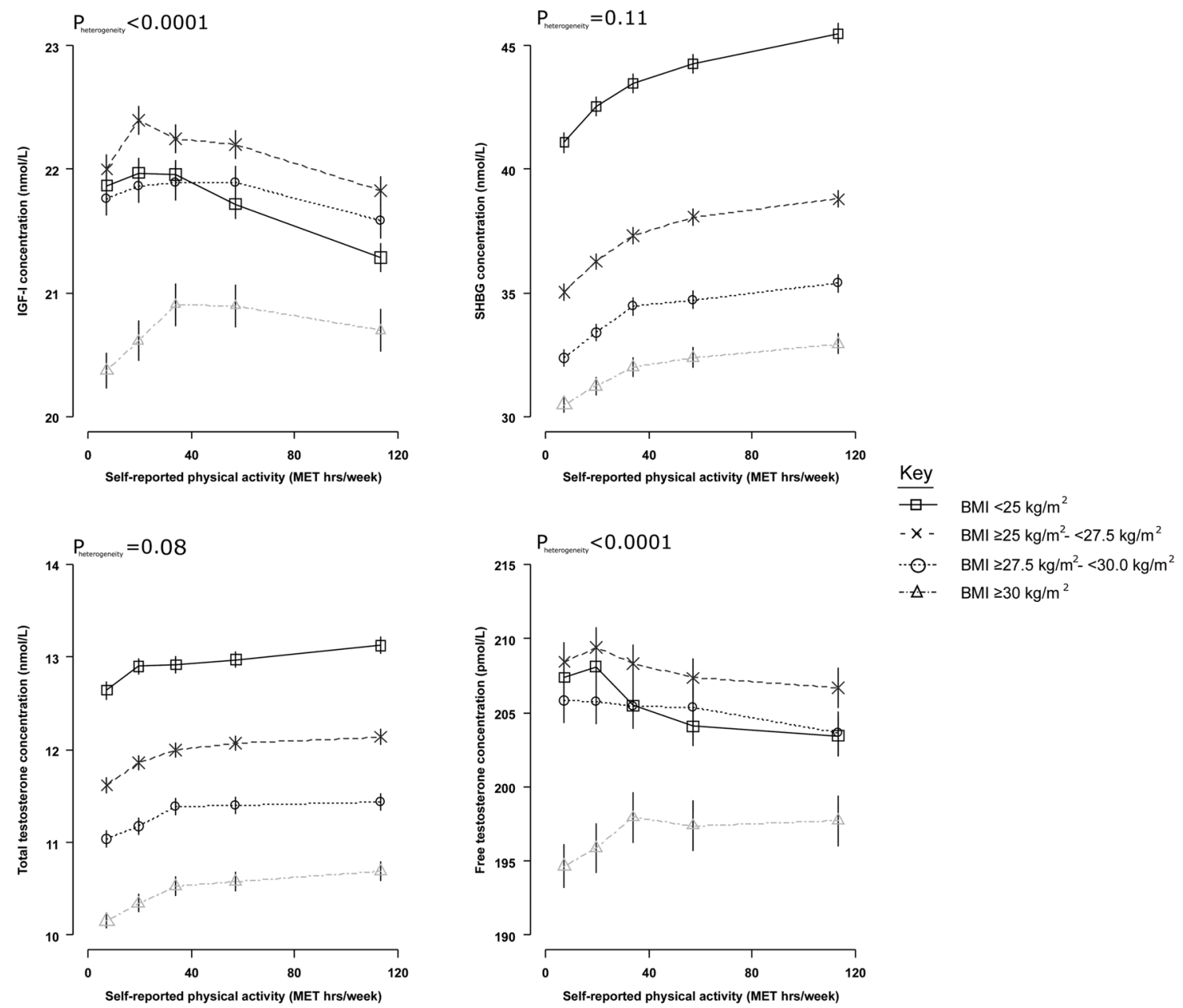

Fig. 3 Adjusted geometric mean circulating hormone concentrations by self-reported physical activity, stratified by categories of BMI, in UK Biobank male participants. Estimated geometric mean concentrations are adjusted for age at recruitment, geographic area, Townsend deprivation score, racial/ethnic group, height, cigarette smoking,

alcohol consumption. Geometric mean concentrations are presented by data points plotted as the median value within each fifth, with their 95\% CIs represented as horizontal lines. $\mathrm{P}_{\text {heterogeneity }}$ is assessed using the $\mathrm{F}$ test. $\mathrm{BMI}=$ body mass index; IGF-I = insulin-like growth factorI; $\mathrm{SHBG}=$ sex hormone-binding globulin

our analysis, the associations with physical activity were attenuated following adjustment for BMI, which may support the role of factors relating to body composition (possibly due to higher physical activity levels [45]), in driving the observed associations of physical activity with hormone concentrations.

Although there was little evidence of a biologically meaningful difference in IGF-I and free testosterone concentrations by physical activity overall, we observed evidence of a positive association among men with obesity, but not among men with a normal BMI. Furthermore, men with obesity who engaged in high levels of measured physical activity had IGF-I and free testosterone concentrations that were similar to non-obese men. Men with obesity on average have higher insulin resistance and low-grade inflammation, so these results may indicate a greater effect of physical activity on these factors [49], possibly normalising hormone concentrations [50, 51]. However, we cannot rule out bias due to better general health in these men. Clinical trials have examined possible associations between physical activity interventions and these hormones in men who are overweight and obese, but results are inconsistent $(n<100)$ [52-54]. Given the small magnitude of the associations observed in this study, larger trials may be necessary to corroborate our findings.

We also observed evidence of heterogeneity in the associations of physical activity with biomarker concentrations by employment status and manual labour at work, although the magnitudes of the associations remained small. This heterogeneity may be due to differences in the types and durations of physical activity that these groups engage in $[55,56]$, related to residual confounding from 
factors such as lifestyle differences between these populations [56] or due to chance.

The magnitudes of the associations between physical activity and hormone concentrations were slightly larger using accelerometer-measured physical activity (without adjustment for BMI). Accelerometers provide objectively measured estimates of physical activity levels and so are less likely to be biased; therefore, the greater magnitude of associations we observed might be due to reduced measurement error. Accelerometers capture general activity throughout the week, whereas self-reported METs are more likely to reflect leisure time activity [57], and consequently these physiological differences might reflect differences in the type of physical activity being captured. Associations were also larger with higher intensities of measured and self-reported physical activity, which may support the hypothesis that higher intensity of physical activity may lead to greater improvements in liver health, particularly lower liver fat $[58,59]$. Therefore, our results do not preclude the possible role of more vigorous physical activity on hormone concentrations, and future research using heart rate monitors may help to elucidate this. Our findings also do not exclude the possible role of acute short-term effects or exercise regimes that may be above those observed in the general population [60].

This analysis has several strengths. It is the largest dataset currently available with hormone measurements and both objectively measured and self-reported physical activity levels, as well as physical activity intensities. The large sample size allows us to estimate moderate associations with greater precision and to detect more modest associations. The comprehensive exposure data enable us to compare associations with hormones using different methods to estimate physical activity and to account for a wide range of possible confounders and health factors. UK Biobank also collected repeat measurement data, which allowed us to verify that our results are robust over an approximately 5-year period.

A limitation of the analysis is that accelerometer-measured physical activity was recorded on average 5.7 years after blood draw, although accelerometer-measured physical activity has been shown to have good reproducibility over the medium term (2-3 years, intraclass correlation $=0.67-0.82$ ) [61]. The UK Biobank participants are predominantly white and are healthier than the sampling population; however, the directions of the associations found here are likely to be generalizable [25, 62]. Although we have taken measures to adjust for confounding factors, residual confounding may still be present, particularly for factors which are related to health and/or body composition, which are difficult to disentangle from physical activity [45].

\section{Conclusion}

In conclusion, the associations of physical activity with IGF-I, SHBG and testosterone were, at most, modest; and following adjustment for BMI, these associations were substantially attenuated. Therefore, it is unlikely that changes in these circulating hormones explain the associations of physical activity with risk of cancer either independently or via BMI.

Supplementary Information The online version contains supplementary material available at https://doi.org/10.1007/s10552-021-01466-6.

Authors' contributions ELW drafted the original manuscript, analysed the data and obtained funding; APC, TJK and RCT supervised the project, and commented on the paper and methodology; AD provided accelerometer variables and commented on the paper and methodology; NEA and STT provided feedback on the manuscript and methodology; GKF was involved in data curation and methodology.

Funding This work is supported by Cancer Research UK (grant numbers C8221/A19170 and C8221/A29017). ELW is supported by the Nuffield Department of Population Health Early Career Research Fellowship. APC is supported by a Cancer Research UK Population Research Fellowship (C60192/A28516) and by the World Cancer Research Fund (WCRF UK), as part of the Word Cancer Research Fund International grant programme (2019/1953). AD is supported by the National Institute for Health Research (NIHR) Oxford Biomedical Research Centre (BRC), the Alan Turing Institute and the British Heart Foundation (grant number SP/18/4/33803), and Health Data Research UK, an initiative funded by UK Research and Innovation, Department of Health and Social Care (England) and the devolved administrations, and leading medical research charities. STT is supported by the Girdlers' New Zealand Health Research Council Fellowship (19/031).The views expressed are those of the author(s) and not necessarily those of the NIHR.

Data availability UK Biobank is an open access resource, and the study website https://www.ukbiobank.ac.uk/ has information on available data and access procedures.

Code availability Code will be made available upon request.

\section{Declarations}

Conflict of interest The authors have no conflicts of interest to disclose.

Ethical approval This research has been conducted using the UK Biobank Resource under Application Number 3282. The UK Biobank study was approved by the North West Multi-Centre Research Ethics Committee (Reference Number 06/MRE08/65).

Consent to participate At recruitment, all participants gave written informed consent to participate and for their health to be followed-up through linkage to electronic medical records. Informed consent was obtained from all individual participants included in the study.

Open Access This article is licensed under a Creative Commons Attribution 4.0 International License, which permits use, sharing, adaptation, distribution and reproduction in any medium or format, as long 
as you give appropriate credit to the original author(s) and the source, provide a link to the Creative Commons licence, and indicate if changes were made. The images or other third party material in this article are included in the article's Creative Commons licence, unless indicated otherwise in a credit line to the material. If material is not included in the article's Creative Commons licence and your intended use is not permitted by statutory regulation or exceeds the permitted use, you will need to obtain permission directly from the copyright holder. To view a copy of this licence, visit http://creativecommons.org/licenses/by/4.0/.

\section{References}

1. Matthews CE, Moore SC, Arem H et al (2019) Amount and intensity of leisure-time physical activity and lower cancer risk. J Clin Oncol 38:686-697

2. Hojman P, Gehl J, Christensen JF, Pedersen BK (2018) Molecular mechanisms linking exercise to cancer prevention and treatment. Cell Metab 27:10-21

3. McTiernan A (2008) Mechanisms linking physical activity with cancer. Nat Rev Cancer 8:205-211

4. Patel AV, Friedenreich CM, Moore SC et al (2019) American college of sports medicine roundtable report on physical activity, sedentary behavior, and cancer prevention and control. Med Sci Sports Exerc 51:2391-2402

5. Major JM, Laughlin GA, Kritz-Silverstein D, Wingard DL, Barrett-Connor E (2010) Insulin-like growth factor-I and cancer mortality in older men. J Clin Endocrinol Met 95:1054-1059

6. Travis RC, Appleby PN, Martin RM et al (2016) A meta-analysis of individual participant data reveals an association between circulating levels of IGF-I and prostate cancer risk. Cancer Res 76:2288-2300

7. Watts EL, Appleby PN, Perez-Cornago A et al (2018) Low free testosterone and prostate cancer risk: a collaborative analysis of 20 prospective studies. Eur Urol 74:585-594

8. Knuppel A, Fensom GK, Watts EL et al (2020) Circulating insulin-like growth factor-I (IGF-I) concentrations and incidence of 30 cancers: prospective analyses in UK Biobank. Cancer research Cancer Res 1281:2020

9. Watts EL, Perez-Cornago A, Knuppel A, Tsilidis KK, Key TJ, Travis RC (2021) Prospective analyses of testosterone and sex hormone-binding globulin with the risk of 19 types of cancer in men and postmenopausal women in UK Biobank. Int J Cancer. https://doi.org/10.1002/ijc.33555

10. Steeves JA, Fitzhugh EC, Bradwin G, McGlynn KA, Platz EA, Joshu CE (2016) Cross-sectional association between physical activity and serum testosterone levels in US men: results from NHANES 1999-2004. Andrology 4:465-472

11. Parekh N, Roberts CB, Vadiveloo M, Puvananayagam T, Albu JB, Lu-Yao GL (2010) Lifestyle, anthropometric, and obesityrelated physiologic determinants of insulin-like growth factor-1 in the third national health and nutrition examination survey (1988-1994). Ann Epidemiol 20:182-193

12. Muller M, den Tonkelaar I, Thijssen JH, Grobbee DE, van der Schouw YT (2003) Endogenous sex hormones in men aged 40-80 years. Eur J Endocrinol 149:583-589

13. Shiels MS, Rohrmann S, Menke A et al (2009) Association of cigarette smoking, alcohol consumption, and physical activity with sex steroid hormone levels in US men. Cancer Causes Control 20:877-886

14. Hawkins VN, Foster-Schubert K, Chubak J et al (2008) Effect of exercise on serum sex hormones in men: a 12-month randomized clinical trial. Med Sci Sports Exerc 40:223-233
15. Winzer BM, Whiteman DC, Reeves MM, Paratz JD (2011) Physical activity and cancer prevention: a systematic review of clinical trials. Cancer Causes Control 22:811-826

16. Nishida Y, Matsubara T, Tobina T et al (2010) Effect of lowintensity aerobic exercise on insulin-like growth factor-I and insulin-like growth factor-binding proteins in healthy men. Int J Endocrinol. https://doi.org/10.1155/2010/452820

17. McTiernan A, Sorensen B, Yasui Y et al (2005) No effect of exercise on insulin-like growth factor 1 and insulin-like growth factor binding protein 3 in postmenopausal women: a 12-month randomized clinical trial. Cancer Epidemiol Biomarkers Prev 14:1020-1021

18. Kumagai H, Zempo-Miyaki A, Yoshikawa T, Tsujimoto $\mathrm{T}$, Tanaka K, Maeda S (2016) Increased physical activity has a greater effect than reduced energy intake on lifestyle modification-induced increases in testosterone. J Clin Biochem Nutr 58:84-89

19. Hayes LD, Herbert P, Sculthorpe NF, Grace FM (2017) Exercise training improves free testosterone in lifelong sedentary aging men. Endocr Connect 6:306-310

20. Watts EL, Appleby PN, Albanes D et al (2017) Circulating sex hormones in relation to anthropometric, sociodemographic and behavioural factors in an international dataset of 12,300 men. PLOS ONE. 12:e187741

21. Watts EL, Perez-Cornago A, Appleby PN et al (2019) The associations of anthropometric, behavioural and sociodemographic factors with circulating concentrations of IGF-I, IGF-II, IGFBP-1, IGFBP-2 and IGFBP-3 in a pooled analysis of 16,024 men from 22 studies. Int J Cancer 145:3244-3256

22. Tin Tin S, Reeves GK, Key TJ (2020) Body size and composition, physical activity and sedentary time in relation to endogenous hormones in premenopausal and postmenopausal women: findings from the UK Biobank. Int J Cancer 147:2101-2115

23. Ev E, Altman DG, Egger M, Pocock SJ, Gøtzsche PC, Vandenbroucke JP (2007) Strengthening the reporting of observational studies in epidemiology (STROBE) statement: guidelines for reporting observational studies. BMJ 335:806-808

24. Sudlow C, Gallacher J, Allen N et al (2015) UK Biobank: an open access resource for identifying the causes of a wide range of complex diseases of middle and old age. PLoS medicine. 12:e1001779

25. Fry A, Littlejohns TJ, Sudlow C et al (2017) Comparison of sociodemographic and health-related characteristics of UK Biobank participants with those of the general population. Am J Epidemiol 186:1026-1034

26. Craig CL, Marshall AL, Sjostrom M et al (2003) International physical activity questionnaire: 12-country reliability and validity. Med Sci Sports Exerc 35:1381-1395

27. Doherty A, Jackson D, Hammerla N et al (2017) Large scale population assessment of physical activity using wrist worn accelerometers: The UK biobank study. PLOS ONE 12:e169649

28. White T, Westgate K, Hollidge $S$ et al (2019) Estimating energy expenditure from wrist and thigh accelerometry in free-living adults: a doubly labelled water study. Int J Obes 43:2333-2342

29. Doherty A, Smith-Byrne K, Ferreira T et al (2018) GWAS identifies 14 loci for device-measured physical activity and sleep duration. Nat Commun 9:5257

30. Willetts M, Hollowell S, Aslett L, Holmes C, Doherty A (2018) Statistical machine learning of sleep and physical activity phenotypes from sensor data in 96,220 UK Biobank participants. Sci Rep 8:7961

31. Elliott P, Peakman TC (2008) The UK Biobank sample handling and storage protocol for the collection, processing and archiving of human blood and urine. Int J Epidemiol 37:234-244

32. Woods P, Owen JM (2008) Designing and implementing a largescale automated $-80^{\circ} \mathrm{C}$ archive. Int J Epidemiol 37:i56-i61 
33. Goldman AL, Bhasin S, Wu FCW, Krishna M, Matsumoto AM, Jasuja R (2017) A reappraisal of testosterone's binding in circulation: physiological and clinical implications. Endocr Rev 38:302-324

34. Bartsch W (1980) Interrelationships between sex hormone-binding globulin and testosterone, 5 alpha-dihydrotestosterone and oestradiol-17 beta in blood of normal men. Maturitas 2:109-118

35. UK Biobank. (2013) Repeat assessment data.

36. UK Biobank. (2019) Biomarker assay quality procedures: approaches used to minimise systematic and random errors (and the wider epidemiological implications).

37. Kalyani RR, Gavini S, Dobs AS (2007) Male hypogonadism in systemic disease. Endocrinol Metab Clin North Am 36:333-348

38. Perez-Cornago A, Key TJ, Allen NE et al (2017) Prospective investigation of risk factors for prostate cancer in the UK Biobank cohort study. Br J Cancer 117:1562-1571

39. Allen NE, Appleby PN, Kaaks R, Rinaldi S, Davey GK, Key TJ (2003) Lifestyle determinants of serum insulin-like growth-factorI (IGF-I), C-peptide and hormone binding protein levels in British women. Cancer Causes Control 14:65-74

40. Devin JL, Bolam KA, Jenkins DG, Skinner TL (2016) The influence of exercise on the insulin-like growth factor axis in oncology: physiological basis, current, and future perspectives. Cancer Epidemiol Bio Prev 25:239-249

41. Zheng G, Qiu P, Xia R et al (2019) Effect of aerobic exercise on inflammatory markers in healthy middle-aged and older adults: a systematic review and meta-analysis of randomized controlled trials. Front Aging Neurosci 11:98

42. Simo R, Saez-Lopez C, Barbosa-Desongles A, Hernandez C, Selva DM (2015) Novel insights in SHBG regulation and clinical implications. Trends Endocrinol Metab 26:376-383

43. Bird SR, Hawley JA (2017) Update on the effects of physical activity on insulin sensitivity in humans. BMJ Open Sport Exerc Med. 2:e000143

44. Wang S-t, Zheng J, Peng H-w et al (2020) Physical activity intervention for non-diabetic patients with non-alcoholic fatty liver disease: a meta-analysis of randomized controlled trials. BMC Gastroenterol 20:66

45. Bradbury KE, Guo W, Cairns BJ, Armstrong MEG, Key TJ (2017) Association between physical activity and body fat percentage, with adjustment for BMI a large cross-sectional analysis of UK Biobank. BMJ Open. 7:e011843

46. Hayes LD, Herbert P, Sculthorpe NF, Grace FM (2017) Exercise training improves free testosterone in lifelong sedentary aging men. Endocrine Connect 6:306

47. van Gils CH, Peeters PHM, Schoenmakers MCJ et al (2009) Physical activity and endogenous sex hormone levels in postmenopausal women: a cross-sectional study in the prospect-EPIC cohort. Cancer Epidemiol Bio Prev 18:377-383

48. Dwyer AA, Quinton R (2019) Anatomy and physiology of the hypothalamic-pituitary-gonadal (HPG) axis. In: Llahana S, Follin C, Yedinak C, Grossman A (eds) Advanced practice in endocrinology nursing. Springer International Publishing, Cham, pp 839-852
49. Shoelson SE, Herrero L, Naaz A (2007) Obesity, inflammation, and insulin resistance. Gastroenterology 132:2169-2180

50. Pitteloud N, Hardin M, Dwyer AA et al (2005) Increasing insulin resistance is associated with a decrease in Leydig cell testosterone secretion in men. J Clin Endocrinol Met 90:2636-2641

51. Thomas GA, Kraemer WJ, Comstock BA, Dunn-Lewis C, Maresh CM, Volek JS (2013) Obesity, growth hormone and exercise. Sports Med 43:839-849

52. Kumagai H, Yoshikawa T, Zempo-Miyaki A et al (2018) Vigorous physical activity is associated with regular aerobic exerciseinduced increased serum testosterone levels in overweight/obese men. Horm Metab Res 50:73-79

53. Armamento-Villareal R, Aguirre LE, Qualls C, Villareal DT (2016) Effect of lifestyle intervention on the hormonal profile of frail, obese older men. J Nutr Health Aging 20:334-340

54. Chen H-T, Chung Y-C, Chen Y-J, Ho S-Y, Wu H-J (2017) Effects of different types of exercise on body composition, muscle strength, and IGF-1 in the elderly with sarcopenic obesity. J Am Geriatr Soc 65:827-832

55. Kwak L, Berrigan D, Van Domelen D, Sjöström M, Hagströmer M (2016) Examining differences in physical activity levels by employment status and/or job activity level: gender-specific comparisons between the United States and Sweden. J Sci Med Sport 19:482-487

56. Coenen P, Huysmans MA, Holtermann A et al (2018) Do highly physically active workers die early? A systematic review with meta-analysis of data from 193696 participants. Br J Sports Med 52:1320

57. Kozey SL, Lyden K, Howe CA, Staudenmayer JW, Freedson PS (2010) Accelerometer output and MET values of common physical activities. Med Sci Sports Exerc 42:1776-1784

58. Kistler KD, Brunt EM, Clark JM et al (2011) Physical activity recommendations, exercise intensity, and histological severity of nonalcoholic fatty liver disease. Am J Gastroenterol 106:460-469

59. van der Windt DJ, Sud V, Zhang H, Tsung A, Huang H (2018) The effects of physical exercise on fatty liver disease. Gene Expr 18:89-101

60. Lane AR, Magallanes CA, Hackney AC (2019) Reproductive dysfunction from exercise training: the "exercise-hypogonadal male condition." Arch Med Deporte 36:319-322

61. Keadle SK, Shiroma EJ, Kamada M, Matthews CE, Harris TB, Lee IM (2017) Reproducibility of accelerometer-assessed physical activity and sedentary time. Am J Prev Med 52:541-548

62. Batty GD, Gale CR, Kivimäki M, Deary IJ, Bell S (2020) Comparison of risk factor associations in UK Biobank against representative, general population based studies with conventional response rates: prospective cohort study and individual participant meta-analysis. BMJ. 368:m131

Publisher's Note Springer Nature remains neutral with regard to jurisdictional claims in published maps and institutional affiliations. 